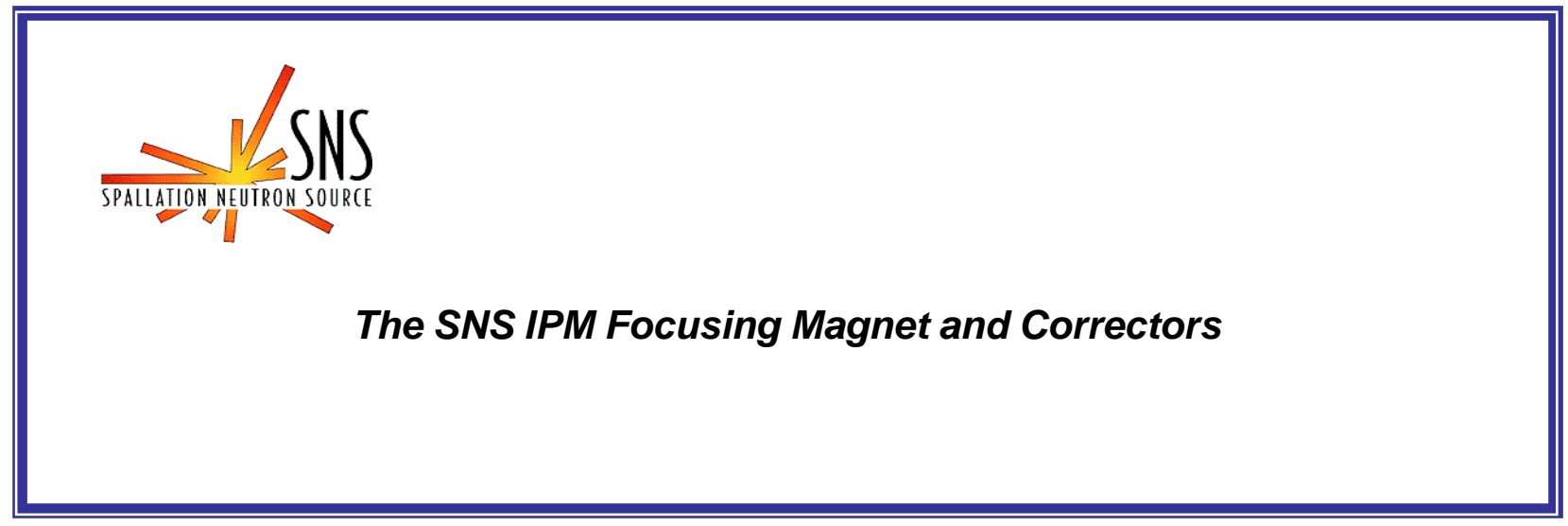

BNL/SNS TECHNICAL NOTE

NO. 127

Roger Connolly

BNL, Upton, NY 11973, USA

September 5, 2003

COLLIDER-ACCELERATOR DEPARTMENT

BROOKHAVEN NATIONAL LABORATORY

UPTON, NEW YORK 11973 


\title{
The SNS IPM Focusing Magnet and Correctors
}

\author{
Roger Connolly
}

This note is to reevaluate the magnet field requirements for the SNS IPM. In the original design tech note [1] we concluded that a field of $0.1 \mathrm{~T}$ was required for accurate profile measurements. As the IPM design has matured it has become clear that a lower field will produce acceptable measurement results and will result in a more simple and less expensive instrument.

The IPM is placed inside the gap of a dipole magnet to constrain the signal electrons to travel perpendicular to the collection plane. To accomplish this task the field must be strong enough to overcome transverse space-charge electric fields and profile broadening caused by the electron recoil momentum from the ionizing collision with the beam particle.

Of these two effects the recoil momentum is the more difficult to overcome. The maximum space charge electric field in the accumulation ring is $10^{5} \mathrm{~V} / \mathrm{m}$. The component of this field perpendicular to the collector plane causes no broadening. The component parallel to the collector plane together with the magnetic field causes the electron to execute cycloidal motion. A field of $0.024 \mathrm{~T}$ restricts the gyration radius to $1.0 \mathrm{~mm}$ for an electron in the strongest transverse space charge field. The collector channels are $3.0 \mathrm{~mm}$ wide.

The spectrum of recoil energies of the electrons is strongly peaked at low energies so that $95 \%$ of signal electrons have energies $<200 \mathrm{eV}$. A field of $0.024 \mathrm{~T}$ confines $200 \mathrm{eV}$ electrons to a Larmor radius of $1.4 \mathrm{~mm}$. Thus with a $0.024 \mathrm{~T}$ magnet over $95 \%$ of signal electrons will be collected on the channel over which they are created.

For these reasons I propose changing the magnet requirement to 250 Gauss. Also I propose that the design return to permanent magnet dipoles. At this low field the risk of damaging multipoles developing from radiation induced demagnetization is greatly reduced.

The magnets will be designed similar to the RHIC magnets and will use ceramic magnet material bricks which are very inexpensive. A single magnet will be designed and four will be built for each IPM. Two will be placed together for the detector and one will be placed on either side for a full three-bump orbit correction. Again, at these low fields, any orbit distortion caused by slightly mismatched magnets will be small and can be taken out with correctors. Since permanent magnets are used the orbit corrections will naturally happen during machine commissioning.

[1] R. Connolly, P. Cameron, T.J. Shea and R. Witkover, Ionization Profile Monitor for SNS, BNL/SNS Tech Note \#059, http://server.ags.bnl.gov/bnlags/bnlsns/sns.html 